\title{
Intervención educativa intercultural para un diálogo de saberes indígena y escolar ${ }^{1}$
}

\section{Intercultural educational intervention for a dialogue of indigenous and school knowledge}

\section{Intervenção educativa intercultural para um diálogo de saberes indígenas e escolares}

\author{
Daniel Quilaqueo Rapimán*
}

\begin{abstract}
RESUMEN
Este artículo plantea la incorporación de episteme indígena al currículum escolar, mediante un método de intervención educativa intercultural con aportes epistémicos de la educación mapuche y escolar. Se analiza, por una parte, que la intervención educativa intercultural implica asumir la igualdad de la condición humana sobre la base de un diálogo de saberes con el fin de garantizar la libertad, la igualdad y la cohesión social; y por otra parte, como desafío sociopolítico para el reconocimiento de la existencia y supervivencia de los pueblos indígenas con sus conocimientos educativos, lenguas e identidades culturales.
\end{abstract}

Palabras clave: Intervención educativa. Interculturalidad. Educación escolar. Educación indígena.

1 Este artículo es financiado por el proyecto FONDECYT N 1181314 "Diálogo de saberes educativos mapuche y escolar: construcción de una base epistémica intercultural de conocimientos" y Proyecto FONDEF ID 16110350 "Modelo de intervención educativa intercultural en contexto indígena".

"Universidad Católica de Temuco. Facultad de Educación. Temuco, Chile. Investigador Asociado del Centre Interuniversitaire d'Études et de Recherches Autochtones (CIÉRA) de l'Universite Laval, Québec, Canadá. E-mail: dquilaq@uctemuco.cl. http://orcid.org/0000-0002-5143-4920. 


\begin{abstract}
This article proposes the incorporation of indigenous episteme to the school curriculum through an intercultural educational intervention method with epistemic contributions of mapuche and school education. It is analyzed, on the one hand, that the intercultural educational intervention implies assuming the equality of the human condition based on the dialogue of knowledge in order to guarantee freedom, equality and social cohesion; and on the other hand, as a sociopolitical challenge for the recognition of the existence and survival of indigenous peoples with their educational knowledge, languages and cultural identities.
\end{abstract}

Keywords: Educational intervention. Interculturality. School education. Indigenous education.

\title{
RESUMO
}

Este artigo propõe a incorporação da episteme indígena ao currículo escolar por meio de um método de intervenção educacional intercultural com contribuições epistêmicas do mapuche e da educação escolar. Analisa-se, por um lado, que a intervenção educativa intercultural implica assumir a igualdade da condição humana com base no diálogo do conhecimento, a fim de garantir a liberdade, a igualdade e a coesão social; e, por outro lado, como um desafio sociopolítico para o reconhecimento da existência e sobrevivência dos povos indígenas com seus conhecimentos educacionais, linguagens e identidades culturais.

Palavras-chave: Intervenção educativa. Interculturalidade. Educação escolar. Educação indígena.

\section{Introducción}

Este artículo plantea la cuestión de intervención educativa intercultural, mediante la incorporación del saber educativo indígena al currículum escolar, para construir un diálogo intercultural entre el saber educativo indígena y el escolar. Aborda la problemática de la educación monocultural de la escuela en contexto indígena, puesto que se observa en la relación educando-educador la ausencia de conocimientos sociales y culturales de los estudiantes de origen indígena. Para ello, se apoya en la existencia de una base epistémica de educación 
del pueblo mapuche ${ }^{2} \mathrm{y}$ en los conceptos "intervención educativa", "intervención educativa intercultural" e "interculturalidad".

En relación a la base epistémica de contenidos educativos mapuche, se advierte que en las tres últimas décadas los investigadores Quilaqueo y Quintriqueo (2017) han sistematizado contenidos educativos, mediante proyectos de investigación ${ }^{3}$, desde el enfoque de construcción social de conocimiento educativo, cuyos resultados se presentan en artículos de revistas científicas y libros.

La intervención educativa se entiende como la gestión intencional del currículum escolar, para la realización de acciones que conducen al logro del desarrollo educativo contextualizado del educando, donde "existe un sujeto agente (educando-educador), existe el lenguaje propositivo (se realiza una acción para lograr algo), se actúa en orden a lograr un acontecimiento futuro (la meta) y los acontecimientos se vinculan intencionalmente" (TOURIÑAN, 2011, p. 283). Por su parte, la intervención educativa intercultural, para los actuales contextos de vida mapuche urbano y rural, es un método para superar la monoculturalidad y el monolingüismo del español que llevan a conflictos epistémicos entre el conocimiento educativo mapuche y el escolar de sujetos que conviven en un mismo espacio educativo y territorial (QUILAQUEO; QUINTRIQUEO; RIQUELME; LONCON, 2016; QUILAQUEO; QUINTRIQUEO, 2017).

El concepto de interculturalidad, por una parte su uso en el contexto indígena, asume principalmente un carácter funcional por parte del Estado en relación al diálogo de personas pertenecientes a culturas diferentes, reconociendo las diferencias sin mayor compromiso entre los sujetos y quedando relegado al discurso oficial de la política pública (TUBINO, 2014; QUILAQUEO; QUINTRIQUEO, 2017). Por otra parte, se observa la propuesta de una perspectiva crítica que considera la interculturalidad como parte del problema que se genera al relacionarse con la diversidad social y cultural indígena, del problema estructural-colonial-racial, que ha reconocido al indígena desde una matriz colonial de poder racializado-jerarquizado, haciéndolos minoría, así como

2 La población indígena, objetivo de la educación intercultural bilingue, es de 1.565.915 habitantes. Es decir, el 9.1\% del total del país (17.207.857); de los cuales 106.729 tienen su residencia en la región del Bío-Bío, 309.952 en La Araucanía, 80.958 en Los Ríos y 209.214 en Los Lagos.

3 Proyectos: 1) FONDECYT N. ${ }^{\circ} 1181314$ "Diálogo de saberes educativos mapuche y escolar: construcción de una base epistémica intercultural de conocimientos"; 2) FONDECT N. ${ }^{\circ} 1140562$ "Construcción social del conocimiento educativo mapuche: doble racionalidad y desafíos para una escolarización intercultural"; 3) FONDECYT 1110677 "Tipificación de los métodos educativos mapuche: Bases para una educación intercultural"; 4) FONDECYT N. ${ }^{\circ}$ 10852934) "Racionalidad del método educativo mapuche desde la memoria social de kimches: fundamentos para una educación intercultural"; 5) FONDECYT N. ${ }^{\circ} 1051039$ "Saberes mapuches y conocimientos educativos vernáculos transmitidos por kimches. Sistematización para una educación intercultural”. 
a sus descendientes (QUILAQUEO; TORRES, 2013; WALSH, 2010). Esto permite plantear la siguiente hipótesis de trabajo: un método de intervención educativa intercultural en el currículum escolar, para contexto indígena, es un proceso formativo planificado que puede entregar a los profesores contenidos culturales, sociales y métodos de las pedagogías indígenas para la enseñanza y el aprendizaje en los distintos niveles de educación básica, educación secundaria y la formación de profesores.

Para el desarrollo de este trabajo, se hace referencia a prácticas educativas en contexto indígena de Nueva Zelanda, Canadá, Estados Unidos, México y Chile. Para el caso chileno, se considera la propuesta del Programa de Educación Intercultural Bilingüe y la base epistémica de conocimiento educativo mapuche revelada en investigaciones empíricas antes citadas. El objetivo es mostrar elementos de esta base epistémica y métodos de la educación mapuche, para una intervención educativa intercultural del currículum escolar de escuelas situadas en contexto mapuche.

\section{Referencia de experiencias de intervención educativa}

En Nueva Zelanda, en el desarrollo social del pueblo maorí, se ha implementado el método Te Kotahitanga, para mejorar los logros de aprendizaje de los estudiantes mediante la práctica del kaupapa (BISHOP; BERRYMAN; CAVANAGH; TEDDY, 2009). El Kaupapa ha sido implementado como una estrategia de resistencia con propuestas para un cambio en la educación escolar. Se basa en la libre determinación dentro de las relaciones no dominantes de interdependencia, y da respuesta a las aspiraciones sociales y culturales del pueblo maorí. Es decir, corregir las disparidades existentes, en el proceso de enseñanza y aprendizaje, entre los estudiantes descendientes de europeos colonizadores y los estudiantes maoríes. Esto implica aprender a enseñar, ya que la adquisición de un nuevo conocimiento involucra una reflexión constante y colaboración en el proceso denominado te kotahitanga, cuyo propósito es lograr la articulación con los conocimientos que ya poseen los estudiantes de origen maorí. Te kotahitanga significa propuesta de colaboración desde una visión común, propósito y meta que los profesores y estudiantes, por separado y en colaboración, pueden promover, vigilar y reflexionar sobre los resultados que a su vez conducen a mejoras en el rendimiento académico (BISHOP; BERRYMAN; CAVANAGH; TEDDY, 2009). Es decir, considerar los elementos no visibles de la sociedad y cultura maorí, como los valores, los modos de comunicación, 
la toma de decisiones y los procesos de resolución de problemas, junto con la visión del mundo y de los procesos de producción del conocimiento que ayudan a los individuos y grupos a construir sus significados.

En Canadá existen centros de investigación que estudian los conocimientos indígenas, es el caso de la Universidad de Ontario (ONTARIO MINISTRY OF EDUCATION, 2015). La Universidad ha promovido en conjunto con el gobierno de Ontario "la educación política provincial", asociada al aprendizaje de los estudiantes indígenas métis e inuit. Para ello, ha creado asociaciones con las comunidades y organizaciones aborígenes, trabajando estrechamente el asesoramiento, la tutoría, la planificación de carreras universitarias y los servicios culturales de sensibilización en los colegios, las universidades e instituciones indígenas. El método utilizado es el acceso a estrategias de la política provincial para tener mejores oportunidades, y está relacionado a crear acciones que permitan a los estudiantes obtener mejores resultados académicos.

En los Estados Unidos, la constitución política del Estado de Hawai tiene como propósito promover el estudio de la cultura, el lenguaje y la historia hawaiana (KENNEDY, 2004; SALAÜN, 2013). Con este fin, el Departamento de Educación estableció el Hawaiian Studies Program y el Hawaiian Language Immersion Program, Ka Papahana Kaiapuni Hawai'i. En el año 2015 se establece la Oficina de Educación de Hawai y una de sus principales tareas es la planificación de la implementación de una nueva política conocida como Nā Hopena A 'o, que tiene por objeto la expansión de la educación hawaiana a través del sistema de educación pública K-12 de Hawái para todos los estudiantes y adultos (SALAÜN, 2013).

En México, existe el proyecto diseño de materiales interculturales bilingües del Laboratorio de Lengua y Cultura del Centro de Investigaciones y Estudios Superiores en Antropología Social (CIESAS). En este laboratorio se generan materiales educativos que buscan fortalecer la Red de Educación Inductiva Intercultural (REDIIN) y aportar a la construcción de una nueva educación intercultural bilingüe para México, en atención a los derechos individuales y colectivos de los pueblos indígenas (BERTELY, 2007; ESTEBAN; BASTIANI, 2010). Sus acciones se dirigen a profesores que se desempeñan en contextos indígenas en el nivel educativo preescolar y primario. Se ofrecen diplomados, talleres y laboratorios que se fundan en el inter-aprendizaje entre profesores indígenas y académicos no indígenas. Estos espacios se destinan a la apropiación y aplicación del Método Inductivo Intercultural, acuñado por Gasché (2008) en la Amazonía peruana, por la Unión de Maestros de la Nueva Educación para México (UNEM) y educadores independientes de Chiapas en colaboración con el CIESAS.

En Chile, el Ministerio de Educación (MINEDUC, 2011) ha implementado el Programa de Educación Intercultural Bilingüe (PEIB), cuya finalidad es el reconocimiento, respeto y la valoración de las culturas de los pueblos indígenas, 
mediante el uso y la conservación de sus idiomas junto al español en las áreas de alta densidad indígena (WILLIAMSON; FLORES, 2015; MINEDUC, 2011; CASEN, 2013). Para el caso mapuche, el PEIB, desde el año 2015, fomenta el desarrollo de competencias de la lengua mapuche (mapunzugun) en los estudiantes con guías educativas para el trabajo del Educador tradicional ${ }^{4}$ en los cursos de primer y segundo año básico. Sin embargo, esta intervención intercultural no cubre todas las asignaturas del currículum escolar.

En síntesis, estas experiencias de intervenciones educativas presentan las siguientes perspectivas: 1) intervención educativa sostenida en métodos educativos propios, con el objetivo de mejorar los aprendizajes mediante la colaboración con la comunidad maorí; 2) intervención educativa principalmente de inmersión lingüística, cuyo objetivo es mejorar los resultados académicos de los estudiantes en el caso de Canadá y Estados Unidos; 3) intervención educativa sostenida en una propuesta de educación intercultural en relación a los derechos individuales y colectivos indígenas, dirigida a profesores que trabajan en contextos indígenas, para el caso de México; y 4) intervención educativa técnico-funcional a la política del Estado para cumplir con las demandas de los pueblos indígenas de Chile, respeto al Convenio 169 de la OIT y a la Ley Indígena nacional.

\section{Característica de la educación escolar en contexto mapuche}

La educación chilena presenta tres particularidades principales: 1) el carácter monocultural y eurocéntrico; 2) el carácter colonial del currículum escolar; y 3) el carácter descontextualizado de la formación de los profesores.

\section{Carácter monocultural de la educación escolar}

La monoculturalidad de la escuela aún tiene como objetivo la homogeneización cultural, mediante el uso de la lengua española, por parte de los grupos no indígenas, primero en los territorios coloniales de la Corona española y poste-

4 El Educador Tradicional es un agente educativo mapuche, reconocido por la comunidad y el Ministerio de Educación, para mediar y facilitar la incorporación de conocimiento mapuche en la asignatura de lengua indígena en conjunto con un profesor mentor. 
riormente en los territorios de los Estados nacionales (SERRANO; PONCE DE LEÓN; RENGIFO, 2012; SALAÜN, 2013). Además, Akkari (2009) plantea que el carácter monocultural de la educación escolar ha tenido las siguientes fuentes de referencias: 1) la educación religiosa y la alfabetización; 2) el positivismo y la herencia filosófica de la Ilustración, orientado a combatir la ignorancia de las personas, conseguir su progreso y emancipación con valores universales; y 3) la consolidación del nacionalismo y la formación del espíritu patriótico de los futuros ciudadanos. Estas tres fuentes han configurado las directrices de la educación escolar que ha sido masificada en todo el mundo para construir sociedades homogéneas (MAMPAEY; ZANONI, 2015). Así, observamos que cada una de estas prácticas ha tenido, en la educación chilena, como finalidad que los estudiantes de grupos indígenas adquiriesen sólo normas, valores y conocimientos de los grupos descendientes de europeos, cuyo resultado es la reproducción del racismo epistémico institucional (TUBINO, 2014).

\section{Carácter colonial del currículum escolar}

El carácter colonial del currículum escolar se expresa en el racismo epistémico del conocimiento educativo mapuche. Este es un tema poco estudiado en el contexto chileno, sin embargo, se observa en un estudio realizado por Cantoni en los años 1960 la percepción y experiencia de trato discriminatorio de mapuches del medio urbano y rural. Revela que el $82 \%$ de los adultos declaraba ser tratado como inferior por los chilenos no indígenas por el hecho de ser mapuche (CANTONI, 1978). Asimismo, en un estudio de los autores Merino, Quilaqueo y Saiz (2008), con una muestra de cien mapuches habitantes de las ciudades de Temuco y Santiago, revela que los temas más recurrentes son discriminación en la educación, discriminación laboral y discriminación en las oficinas de servicios públicos. Es decir, los mapuches son conscientes del hecho de sufrir discriminación y cuyas raíces están en la educación monocultural y monolingüe del currículum escolar. Puesto que el carácter monocultural de la escuela, tanto en Chile como en la mayoría de los países latinoamericanos, reposa en el pasado colonial de la Corona Española; lo que posteriormente el Estado-nación, con el apoyo de los colonos, continuó con el método de disciplinamiento de los hijos de los indígenas en internados de misiones religiosas (BOUSQUET, 2012; SALAÜN, 2013; QUILAQUEO; QUINTRIQUEO, 2017; MANSILLA; LLANCAVIL; MIERES; MONTANARES, 2016). 
Así, el Estado-nación construyó una cultura escolar influida por el discurso de la colonización de los territorios indígenas y de sus habitantes, desconociendo cualquier saber diferente del saber escolar (BONFIL, 1977; KRIEKEN, 1999; KENNEDY, 2004; MUNROE; BORDEN; MURRAY; TONEY; MEADER, 2013). Sin embargo, también existen discursos de reivindicaciones y propuestas indígenas sobre la jerarquización social y cultural que los discrimina y excluye (FOERSTER; MONTECINO, 1988; QUILAQUEO; QUINTRIQUEO, 2017). En Chile, los dirigentes indígenas han planteado ajustar el sentido de las prácticas educativas a la diversidad social y cultural, con acciones que cambiasen la relación escuela-familia-comunidad (QUILAQUEO; TORRES, 2013). El propósito, explícito e implícito, ha sido superar las tensiones epistémicas, culturales y sociales que históricamente se han producido entre lo que entrega el currículum escolar y las necesidades de educación escolar contextualizada que los padres de familia han planteado por medio de los dirigentes de sus comunidades; por ejemplo, la petición de profesores formados con las epistemes educativas mapuche (QUILAQUEO; QUINTRIQUEO, 2017).

\section{Carácter descontextualizado de la formación de los profesores}

La monoculturalidad de la educación en la formación de profesores tiene sus orígenes en la creación de los sistemas educativos modernos del siglo XIX, apoyada en la cultura occidental eurocéntrica (HARRIS, 2002; QUINTRIQUEO; MCGINITY, 2009; MANSILLA; LLANCAVIL; MIERES; MONTANARES, 2016). Se observa que la formación de profesores en las universidades, en Chile, no considera el conocimiento social y cultural indígena en sus planes y programas de formación. Esto lleva a que en su desempeño profesional "pueden transmitir sus propios prejuicios, no sólo a través de sus comentarios y comportamientos, sino también mediante el establecimiento de un clima de intolerancia y etnocentrismo en el aula" (PORTERA, 2014, p. 166). Es decir, al no poseer la formación para aprender a conocer y comunicarse en contextos sociales y culturales indígenas, desconocen cómo combatir el monoculturalismo, el racismo y el etnocentrismo (QUILAQUEO; TORRES, 2013). En este sentido, se observa que las prácticas pedagógicas, en contexto mapuche, es un ámbito permeado por un racismo epistemológico. Es decir, que la cultura escolar construida como uno de los principales medios para dominar a los grupos indígenas, ha provocado la exclusión de sus epistemologías (SCHEURICH; YOUNG, 1997; QUILAQUEO; QUINTRIQUEO; RIQUELME; LONCON, 2016). Las 
consecuencias tienen relación con los antagonismos sociales, culturales, políticos, económicos y educativos, puesto que se observa en la invisibilización y minorización tensionada por aspectos políticos y económicos (PINTO, 2003; QUILAQUEO; TORRES, 2013).

En efecto, para contrarrestar la exclusión de las epistemologías de la educación indígena, se observa que un enfoque teórico y práctico de interculturalidad crítica del currículum escolar posibilita el conocimiento de los sujetos con culturas diferentes desde otras epistemologías para lograr el respeto a la diversidad y la consolidación de los derechos civiles (OLVEIRA; RODRÍGUEZ; GUTIÉRREZ; TOURIÑÁN, 2009). Es decir, una educación intercultural con pertinencia social y cultural requiere innovar la formación de profesores en los siguientes aspectos: 1) comprender las relaciones sociales de los estudiantes que pertenecen a distintas sociedades y culturas; 2) participación en la co-construcción de los programas educativos; 3 ) reelaboración de instrumentos educativos orientados a superar los prejuicios raciales; y 4) consideración de la relación entre la escuela, familia y comunidad en los proyectos educativos, en coherencia con las epistemes de los pueblos indígenas.

Comprender la interculturalidad desde un enfoque de intervención educativa intercultural, que requiere reforzar la formación profesional en valores, el aprendizaje cooperativo en redes y el reconocimiento de los pueblos indígenas. Puesto que, al referirnos a lo intercultural, estamos considerando un posicionamiento en el contexto donde actuamos, en relación a la realidad social y la complejidad cultural resultante de la carga monocultural-monolingüe. Así, el enfoque central de la intervención educativa es la reciprocidad e el intercambio con la escuela-familia-comunidad, porque permite reconocer y desarticular la tradición de la asimilación que induce el currículum escolar. Para ello, es necesario una política educativa que considere el conocimiento de los pueblos indígenas en relación al territorio, los recursos naturales y métodos educativos con fines sociales para la vida de las personas (QUILAQUEO; QUINTRIQUEO, 2017; GASCHÉ, 2008).

Asimismo, lo intercultural obliga considerar el aprendizaje de los estudiantes tanto individualizado como en colaboración con la flexibilidad de los planes de estudios en los que no sólo se evalúe lo deseable desde la cultura escolar, sino que integre de manera inductiva las dimensiones sociales y culturales relevantes para la familia y su comunidad. Desde este punto de vista, es necesario repensar la educación intercultural, con mayor énfasis, desde la sociedad indígena con sus pedagogías, puesto que "la escolarización ha situado a la educación intercultural en el ámbito de lo cognitivo, como si se tratara de conocer, comprender y respetar las ideas, creencias, tradiciones y lengua de una comunidad..." (ORTEGA, 2001, p. 71), sin reconocer, por ejemplo, las 
tensiones resultantes de la discriminación y el racismo explícito e implícito hacia las personas en los textos y contextos del medio escolar, así como las tensiones políticas y económicas (PINTO, 2003; QUILAQUEO; MERINO; SAIZ, 2007; QUILAQUEO; TORRES, 2013). Puesto que la educación intercultural, en contexto indígena, tanto en el plano cultural como cognitivo, hace abstracción de sus conocimientos y relega los estudiantes de origen indígena a la dominación social y cultural de la sociedad chilena.

Sin embargo, considerar a los pueblos indígenas significa ir más allá del respeto de la cultura del otro, puesto que implica, más bien, la aceptación y acogida de la persona perteneciente a grupos sociales con un fuerte arraigo a sus territorialidades históricas (QUILAQUEO, 2007). Esto lleva a plantear que en la intervención educativa intercultural el académico de formación docente debe promover el respeto y fortalecimiento del estudiante de origen indígena y el grupo al cual pertenece, mediante, al menos, tres pasos: 1) el conocimiento con respeto a él mismo y sus estudiantes, delimitando sus marcos de referencia como portador y conocedor de culturas diferentes; 2) el reconocimiento del significado de la territorialidad indígena; y 3) la mediación para negociar y asegurar los intercambios de puntos de vista y llegar a un diálogo de saberes con aportes epistémicos de los pueblos indígenas y de los planes y programas de formación de profesores (QUILAQUEO; TORRES, 2013).

\section{Aportes epistémicos de la educación mapuche para el currículum escolar}

Los resultados de las investigaciones de los autores Quilaqueo y Quintriqueo (2017) evidencian la coexistencia de lógicas del conocimiento mapuche y occidental, entre los mapuches, identificada como doble racionalidad educativa (QUILAQUEO; QUINTRIQUEO; RIQUELME; LONCON, 2016). No obstante, la escuela constituye un espacio social y cultural donde los estudiantes de ascendencia mapuche experimentan la ausencia de sus saberes en el currículum escolar. Sin embargo, los resultados de investigación sobre educación mapuche revelan el concepto kimeltuwün (acción educativa mapuche) como marco epistémico de contenidos, procedimientos y actitudes para la intervención educativa intercultural (QUILAQUEO, 2007; QUILAQUEO; QUINTRIQUEO; TORRES; MUÑOZ, 2014).

El kimeltuwün es una acción educativa que permite el aprendizaje-enseñanza, propio del contexto social y cultural, desarrollado en la familia y 
comunidad entre dos o más personas, para la formación de niños y jóvenes. Se desarrolla en seis etapas: 1) se inicia con un zugu, es decir una idea o interés que prepara al aprendiz para lograr ordenar una idea o comprensión en el marco general de rakizuam (razonamiento); 2) continúa con el zuam y un razonamiento que ordena la idea inicial para su comprensión; 3) sigue con tukulpa zugu, es decir, expresando ideas de un tema deseable de ser aprendido recurriendo a conocimientos específicos alojados en la memoria social de la familia y de la colectividad mapuche histórica; 4) en esta etapa se resitúa el contenido educativo entre los sujetos, haciendo konünpa zugu, es decir, con las primeras explicaciones acerca de los contenidos seleccionados como comienzo de un diálogo de saberes; 5) en esta etapa se evalúa mediante el günezuam (metacognición) el conocimiento construido en la etapa anterior, donde los sujetos en aprendizaje desarrollan la capacidad de cuestionar y cuestionarse progresivamente sobre los contenidos educativos; y 6) kimün representa el resultado de la construcción de un nuevo conocimiento o la comprensión del aprendiz y de quien enseña, lo que constituirá un nuevo zugu de la acción educativa kimeltuwün (QUILAQUEO; QUINTRIQUEO, 2017).

Asimismo, entre las metodologías del conocimiento educativo mapuche se revelan las siguientes: 1) el inatuzugu es el proceso de búsqueda e indagación en el conocimiento alojado en la memoria social de la familia heredada de la colectividad mapuche histórica, cuya finalidad es la comprensión de procesos, hechos, sucesos para interpretarlos desde una racionalidad propia; 2) el nampülkan es la racionalización y representación de la memoria social, cuya finalidad es comprender la realidad social y cultural de un sujeto, desde la propia experiencia del aprendiz y su confrontación con otros conocimientos; y 3) el zapilüwün se refiere a la necesidad de cultivar un saber con otro sujeto, contribuyendo al aprendizaje y a la comprensión del desarrollo de las cosas y de las personas; lo que conlleva intereses y contenidos del contexto cultural de los individuos y grupos sociales (QUILAQUEO; QUINTRIQUEO, 2017).

En relación a los principales procedimientos de enseñanza-aprendizaje encontramos los siguientes: 1) el gübam (consejo educativo) es un aprendizaje-enseñanza desde la costumbre familiar, es intencionado para aumentar progresivamente el conocimiento social y cultural de los niños y adolescentes sobre la base de saberes educativos propios y ajenos; 2) el wewpin (discusión y contraposición de ideas, saberes y conocimientos) se refiere a una técnica discursiva cuyo objetivo es lograr una síntesis, con los mejores argumentos latentes en la memoria social y de los sujetos que realizan el wewpin; 3) el wixankontuwün (visita educativa) es un tipo de relación social donde el sujeto se expone al reconocimiento de los familiares y de la comunidad, cuyo fin es aprender a respetar y recibir el afecto familiar; 4) el nüxam (conversación), 
mientras método, es una conversación formativa cuyo fin es lograr aprender (o enseñar) sobre el parentesco, la historia familiar y de la comunidad, teniendo como fuente de información la memoria social del grupo familiar; y 5) el pentukuwün (saludo) es un saludo entre dos o más personas de la comunidad, o bien de otras territorialidades, con el fin de intercambiar ideas sobre ellos en relación al reconocimiento de los familiares por la línea paterna denominada küpan y por la línea materna tuwün (QUILAQUEO; FERNÁNDEZ; QUINTRIQUEO, 2017).

\section{Discusión y conclusión}

La falta de contenidos y métodos educativos indígenas en el currículum escolar, como asimismo en los planes y programas de formación de profesores de las universidades chilenas, es la base del problema que presenta una intervención educativa intercultural en contexto indígena. En efecto, esto es heredado de la matriz cultural colonial que implícitamente sustenta el currículum escolar monocultural. Puesto que los profesores, al no contar con un currículum escolar que incorpore los conocimientos educativos indígenas, tampoco en los planes y programas de formación en las carreras de pedagogía de las universidades, deben trabajar siguiendo el currículum escolar monocultural.

Por otra parte, se observa que las investigaciones empíricas sobre la construcción social del conocimiento educativo mapuche, mediante el método de co-construcción y co-escritura, con padres de familia y sabios, han revelado una base epistémica de contenidos educativos para una intervención educativa intercultural (QUILAQUEO; SAN MARTIN, 2008; QUILAQUEO; TORRES, 2013; QUILAQUEO; QUINTRIQUEO, 2017). También revelan contenidos pedagógicos desde un discurso propio y crítico, de los padres de familia, en relación a la educación escolar y la cuestión de objetivos pedagógicos de reparación histórica, a través de la incorporación del saber educativo mapuche al currículum escolar. Sin embargo, el argumento de reparación histórica de disminuir el colonialismo cultural en el aula no es reconocido (MAMPAEY; ZANONI, 2015). Es decir, no se reconoce ejercer el derecho a la instrucción con ambas epistemes: educación escolar y educación de los pueblos indígenas. De esta forma, el objetivo señalado por los mapuches es la formación mediante ambas epistemes para contrarrestar el estigma de la monoculturalidad y el monolingüismo de la lengua española.

Luego, la base epistémica de conocimiento educativo indígena, y mapuche en particular, posibilita educar para superar el estigma de la monoculturalidad, 
desde el currículum escolar, con un método de intervención educativa intercultural en escuelas de contextos indígenas (QUILAQUEO; SAN MARTIN, 2008; QUILAQUEO; QUINTRIQUEO, 2017). Para ello, se reconoce que "la marginación del conocimiento indígena debe ser debatida y su relación con el conocimiento universalizado debe ser renegociada" (CONNELL, 2006, p. 91). Así, en la propuesta de intervención educativa intercultural, para el caso mapuche, se observa que la renegociación invita incorporar sus epistemes, puesto que está presente en todas las escuelas situadas en contexto rural y urbano. Para eso, es necesario reconocer la pluralidad epistemológica de los conocimientos, es decir, la construcción de conocimiento que no esté sustentada sólo en la racionalidad científica (OLIVÉ, 2009), porque “el saber indígena es un saber dinámico que se recrea a diario en los actos, hechos y circunstancias del hombre en relación con lo divino, la naturaleza, la familia, la comunidad y la sociedad en general" (JAMIOY, 1997, p. 66).

Los conocimientos educativos indígenas son construcciones sociales que mantienen los elementos culturales del pasado y, a su vez, reflejan los cambios producidos en las relaciones sociales y culturales con la sociedad occidental. Esto se explica en lo que se ha denominado doble racionalidad educativa, sustentada en la base epistémica mapuche y la base epistémica del saber escolar (QUILAQUEO; QUINTRIQUEO; RIQUELME; LONCON, 2016). Es decir, sustentada en bases epistémicas diferentes, porque la base epistémica de conocimiento educativo mapuche tiene como fundamento el conocimiento alojado en la memoria social de los miembros de las comunidades (padres, abuelos y en particular de sabios). Por lo tanto, la base epistémica del saber escolar es una síntesis sobrevalorada del conocimiento científico en relación a la base epistémica de los saberes de los pueblos indígenas (ROCKWELL, 1995; SCHMELKES, 2006; QUILAQUEO; QUINTRIQUEO, 2017).

Por otra parte, se observa que los contenidos y métodos educativos identificados en la base epistémica mapuche se pueden incorporar a la escuela desde una perspectiva crítica de la interculturalidad hacia los pueblos indígenas (WALSH, 2010; TUBINO, 2014), mediante un proceso de co-construcción de conocimiento entre profesores de la escuela, padres de familia y sabios de la comunidad, con una metodología y pedagogía mapuche de contextualización curricular. El propósito es modificar los métodos tradicionales de la escuela, mediante contenidos presentados a los estudiantes, puesto que la lógica de conocimiento de un sujeto implica la autoeducación del educador, respetando la condición de sujeto-agente en el educando, donde la finalidad es lograr una meta que ha sido trazada intencionalmente (TOURIÑAN, 2011). Así, la intervención educativa, desde una perspectiva pedagógica, consiste en un objetivo que permite 
sostener, estimular y modificar las relaciones educando-educador, con el fin de lograr un trabajo efectivo de los estudiantes (LACOURSE; MAUBANT, 2009).

En su dimensión didáctica, la intervención educativa significa una acción planificada y fundamentada epistemológicamente, cuyo propósito es buscar una mejor calidad de los aprendizajes (OLIVA, 1996). Así, la acción planificada implica la existencia de un objeto de colaboración fundado en una unidad de base o un contenido común, un problema a resolver en la educación que se realiza en un contexto específico (LENOIR; LAROSE; DEAUDELIN; KALUBI; ROY, 2002). Desde un punto de vista epistemológico, una unidad de base corresponde a un contenido específico de estudio, a partir del cual se focaliza y se construye un dominio del saber mediante un conjunto de actividades cognoscitivas (LEGENDRE, 1993).

Ahora, subrayando el ámbito pedagógico-intercultural, la intervención educativa significa formar en actitudes y valores a los estudiantes sobre la base de conocimiento y el respeto de las distintas sociedades y manifestaciones culturales, para lograr que la diversidad y las diferencias sean aspectos enriquecedores en la educación escolar. Sin embargo, con frecuencia se tiende a pensar la intervención educativa intercultural como un proceso que atañe solamente a los sujetos que viven en condición de minoría, como es el caso de las comunidades mapuche. No obstante, es un proceso que también requiere cambios y acciones de todos los grupos sociales de un país. Puesto que el valor clave de la educación intercultural es la dignidad de la persona junto a otros valores como la libertad, la igualdad, solidaridad y el respeto (MUÑZSEDANO, 1997; GUERRERO-PACHECO, 2009). Por lo tanto, debe comenzar desde los primeros momentos de la etapa escolar, reconociendo, por ejemplo, la tragedia de los pueblos indígenas, producto de la invasión y pérdida de sus territorios (TODOROV, 1991). Es decir, superar los estereotipos y prejuicios que los adultos de origen no indígena, mediante la educación monocultural, han transmitido; tales como indio, flojo, pobre, borracho, entre otros (MERINO; QUILAQUEO; SAIZ, 2008).

En síntesis, para superar la monoculturalidad y el monolingüismo del español, de la escuela, con la intervención educativa intercultural en contexto indígena, se propone lo siguiente: 1) colaboración sobre la base de un proyecto educativo que considere los objetivos educativos e intereses de la familia y la comunidad, así como los objetivos propios de la educación escolar; 2) contextualización sistémica de la colaboración en situaciones específicas, emergentes en el currículum escolar con el fin de lograr la pertinencia y coherencia del proyecto educativo; 3) participación y relación intercultural consensuada sobre la base e iniciativas que generen el diálogo de saberes en las prácticas educativas; 4) formación y reflexión de reparación con acciones que permitan una sistematización 
de las posibilidades y limitaciones de la intervención educativa; 5) acciones educativas donde los profesores establezcan una relación con las prácticas sociales y culturales del medio familiar y comunitario; 6) compromiso con la calidad de la educación, donde la escuela se asume como una organización donde se aprende, desde un diálogo intercultural entre los actores de la comunidad educativa; y 7) dispositivos de evaluación y seguimiento que permitan alcanzar tanto los objetivos pedagógicos indígenas como los objetivos pedagógicos escolares desde la relación intercultural entre los actores del medio escolar en contexto indígena (LAURENCE, 1992; OLIVA, 1996; PERRENOUD, 2002; GATHER; OLIVIER, 2010; QUILAQUEO; QUINTRIQUEO; TORRES; MUÑOZ, 2014).

Por fin, la intervención educativa intercultural implica asumir la igualdad de la condición humana sobre la base de un diálogo mutuo y la relación de intercambio con el fin de garantizar la libertad, la igualdad y la cohesión social interculturizando el currículum escolar. Su propósito es lograr que los futuros ciudadanos - de origen indígena y no indígena - participen de la sociedad en aspectos políticos, económicos y educativos, entre otros, con conocimientos de la sociedad y la cultura en la cual viven. Esto implica un desafío socioeducativo y sociopolítico por el reconocimiento de la existencia y convivencia con los pueblos indígenas y sus conocimientos educativos, lenguas e identidades, juntamente con sus derechos sociales. Puesto que la intervención educativa intercultural implica un currículum escolar que salga al encuentro de los propósitos monoculturales implícitos y explícitos de la educación escolar, se apuesta en un aprendizaje con los pueblos indígenas, desde la construcción de un enfoque intercultural crítico de la educación, junto a los profesores que trabajan el currículum escolar y académico que forma pedagogos.

\section{REFERENCIAS}

AKKARI, A. Introduction aux approches interculturelles en éducation. Genève: Carnets des Sciences de L'Éducation, 2009.

BERTELY, M. (Coord.). Los hombres y las mujeres de maiz. Democracia y derecho indigena para el mundo. México: Fondo Editorial de la PUCP, Fundación Ford, UNEM, CIESAS, 2007.

BISHOP, R.; BERRYMAN, M.; CAVANAGH, T.; \& TEDDY, L. Te Kotahitanga: Addressing educational disparities facing Maori students in New Zealand. Teaching and Teacher Education, 30, 1-9, 2009. 
BONFIL, G. La declaración de Barbados II y comentarios en Nueva Antropología. Revista de Ciencias Sociales, 7,109-125. México: Universidad Nacional Autónoma de México, 1977.

BOUSQUET, M. P. Êtres libres ou sauvages à civiliser? L'éducation des jeunes Amérindiens dans les pensionnats indiens au Québec, années 1950 à 1970. Revue d'histoire de l'enfance irrégulière, 14, 162-192. Francia: Universitaires de Rennes, 2012.

CANTONI, W. Relaciones del mapuche con la sociedad nacional chilena. En: UNESCO (Ed.), Raza y Clase en la Sociedad Postcolonial, 227-334. Madrid: UNESCO, 1978.

CASEN. Encuesta de Caracterización Socioeconómica. Santiago de Chile: MIDEPLAN, 2013.

CONNELL, R. Conocimiento indígena y poder global: lecciones de los debates africanos. Revista Nómadas, 25, 86-97, 2006.

ESTEBAN, M.; BASTIANI, J. ¿Puede un modelo educativo intercultural combatir la discriminación y la xenofobia? Athenea Digital, 17, 3-16, 2010.

FOERSTER, R.; MONTECINO, S. Organizaciones, lideres y contiendas Mapuches. Santiago: Centro Ecuménico Diego de Medellín; Centro de Estudio de la Mujer, 1988.

GASCHÉ, J. La motivación política de la educación intercultural indígena y sus exigencias pedagógicas. ¿Hasta dónde abarca la interculturalidad? En: BERTELY, M.; GASCHÉ, J. y PODESTÁ, R. (Coords.). Educando en la diversidad. Investigaciones y experiencias educativas interculturales y bilingües. Quito, Ecuador: Abya-Yala, 367-397, 2008.

GATHER, M.; OLIVIER, M. La organización del trabajo escolar. Una oportunidad para pensar la escuela. Barcelona: Editorial GRAÓ, 2010.

GUERRERO-PACHECO, A. Pedagogía intercultural aplicada a la etapa de educación infantil. Málaga: Ediciones Didácticas y Pedagógicas, S. L, 2009.

HARRIS, H. Coyote goes to school: The paradox of Indigenous higher education. Canadian journal of native education, 2, (26), 96-187. Canadá: University of Alberta, 2002.

JAMIOY, J. Los saberes indígenas son patrimonio de la humanidad. Nómada, 7, 64-72, 1997.

KENNEDY, R. The affective work of stolen generations testimony: From the archives to the classroom. Biography, 1, (27), 48-77. Estados Unidos: University of Hawai' $i, 2004$.

KRIEKEN, R. The barbarism of civilization: cultural genocide and the 'stolen generations. The British journal of sociology, 2, (50), 297-315. England: London School of Economics, 1999.

LACOURSE, F.; MAUBANT, P. L'intervention éducative: un cadre conceptuel pertinent. Nouveaux cahiers de la recherche en éducation, 1, (12), 3-8, 2009.

LAURENCE, C. La didactique en questions. Paris: Hachette Édition, 1992. 
LEGENDRE, R. Dictionnaire actuel de l'éducation. Montréal: Guérin éditeur, 1993.

LENOIR, Y.; LAROSE, F.; DEAUDELIN, C.; KALUBI, J-C.; ROY, G-R. L'intervention éducative: clarifications conceptuelles et enjeux sociaux. Pour une reconceptualisation des pratiques d'intervention en enseignement et en formation à l'enseignement. Revue électronique de sociologie Esprit critique, 4, (4), 2002.

MAMPAEY, J.; ZANONI, P. Reproducing monocultural education: ethnic majority staff's discursive constructions of monocultural school practices. British Journal of Sociology of Education, (ahead-of-print), England: London School of Economics, 2015.

MANSILLA, J.; LLANCAVIL, D.; MIERES, M.; MONTANARES, E. Instalación de la escuela monocultural en la Araucanía, 1883-1910: dispositivos de poder y Sociedad Mapuche. Revista Educação y Pesquisa, 42(1), 213-228, 2016.

MERINO, M. E.; QUILAQUEO, D.; SAIZ, J. Una tipología del discurso de discriminación percibida en mapuches de Chile. Revista Signos, 41, (67), 279-297, 2008.

MINEDUC (Ministerio de Educación de Chile). Estudio sobre la implementación de la Educación Intercultural Bilingüe. Santiago de Chile: Programa de Educación Intercultural Bilingüe, 2011.

MUÑOZ-SEDANO, A. Educación Intercultural. Teoría y Práctica. Madrid: Escuela Española, 1997.

MUNROE, E.; BORDEN, L.; MURRAY, A.; TONEY, D.; MEADER, J. Decolonizing Aboriginal education in the 21st century. McGill Journal of Education/Revue des sciences de l'éducation de McGill, 2, (48), 317- 338. Canadá: Universidad McGill, 2013.

OLIVA, J. Crítica de la razón didáctica materiales para el diseño y desarrollo del currículum. Madrid: Editorial Playor, 1996.

OLVEIRA, M.; RODRÍGUEZ, A.; GUTIÉRREZ, M.; TOURIÑÁN, J. Modelos Interculturales. Cuestiones conceptuales para el desarrollo de estrategias de intervención. Revista en Educación, 2, 1-15, 2009.

OLIVÉ, L. Por una auténtica interculturalidad basada en el reconocimiento de la pluralidad epistemológica. En: OLIVÉ, L. et al., Pluralismo Epistemológico, pp.191-218. La Paz, Bolivia: Muela del Diablo, 2009.

ONTARIO MINISTRY OF EDUCATION. Ontario First Nation, Métis, and Inuit Education Policy Framework. Revisado el 15 de abril de 2017. Disponible en: http://www. edu.gov.on.ca/eng/aboriginal/fnmiFramework.pdf. Revisado el 15 de abril de 2017.

ORTEGA, P. La educación moral del ciudadano de hoy. Buenos Aires, Paidós, 2001.

PERRENOUD, P. Les conceptions changeantes du curriculum prescrit: hypothèses. Éducateur, 1, 48-52, 2002.

PINTO, J. La formación del Estado y la Nación, y el pueblo mapuche: De la inclusión a la exclusión. Santiago: DIBAM, 2003. 
PORTERA, A. Intercultural Competence in education, counselling and psychotherapy. Intercultural Education, 2, (25), 157-174. Londres: T\&F for the International Association for Intercultural Education (IAIE), 2014.

QUILAQUEO, D. Saberes y conocimientos indígenas en la formación de profesores de educación intercultural. Educar em Revista, n. 29, 223-239, 2007.

QUILAQUEO, D.; FERNÁNDEZ, C.; QUINTRIQUEO, S. Tipos discursivos a la base de la educación familiar mapuche. Universum, v. 32, n. 1, 2017.

QUILAQUEO, D.; MERINO, M. E.; SAIZ, J. L. Representación social mapuche e imaginario social no mapuche de la discriminación percibida. Atenea, 2007, n. 496, pp. 81-103.

QUILAQUEO, D.; QUINTRIQUEO, S. Métodos educativos mapuche: retos de la doble racionalidad educativa: Aportes para un enfoque de educación intercultural. Ediciones Universidad Católica de Temuco, 2017.

QUILAQUEO, D.; QUINTRIQUEO, S.; RIQUELME, E.; LONCON, E. Educación mapuche y educación escolar en la Araucanía: ¿doble racionalidad educativa? Cadernos de Pesquisa, v. 46, n. 162, 1050-1070, 2016.

QUILAQUEO, D.; QUINTRIQUEO, S.; TORRES, H.; MUÑOZ, G. Saberes educativos mapuches: aportes epistémicos para un enfoque de educación intercultural. Chungara Revista de Antropología Chilena, v. 46, n. 2, 271-283, 2014.

QUILAQUEO, D.; SAN MARTÍN, D. Categorización de saberes educativos mapuche mediante la teoría fundamentada. Estudios pedagógicos, XXXIV, n. 2: 151-168, 2008.

QUILAQUEO, D.; TORRES, H. Multiculturalidad e interculturalidad: desafíos epistemológicos de la escolarización desarrollada en contextos indígenas. Revista ALPHA, 37, 285-300, 2013.

QUINTRIQUEO, S.; MCGINITY, M. Implicancias de un modelo curricular monocultural en la construcción de la identidad sociocultural de alumnos mapuches de la IX Región de la Araucanía, Chile. Estudios Pedagógicos, 35, 173-188, 2009.

ROCKWELL, E. La escuela cotidiana. México: Fondo de Cultura Económica, 1995.

SALAÜN, M. Décoloniser l'école? Hawai'i, Nouvelle-Calédonie. Expériences contemporaines. Collection Essais, 2013.

SCHEURICH, J.; YOUNG, M. Coloring epistemologies: Are our research epistemologies racially biased? Educational Researcher, 26, (4), 4-16, 1997.

SCHMELKES, S. El conocimiento campesino. Revista Mexicana de Investigación Educativa, 28, (11), 333-337, 2006.

SERRANO, S.; PONCE DE LEÓN, M.; RENGIFO, F. Historia de la educación en Chile (1810-2010). Tomo I: Aprender a leer y escribir (1810-1880). Santiago de Chile: Taurus, 2012. 
TODOROV, T. Nosotros y los otros. Reflexión sobre la diversidad humana. México D.F.: Editorial Siglo XXI, 1991.

TOURIÑAN, J. Intervención educativa, Intervención Pedagógica y Educación: La Mirada Pedagógica. Revista Portuguesa de Pedagogía, (Extra-Serie) 283-307, 2011.

TUBINO, F. El trasfondo epistémico de los conflictos interculturales. Revista Contextualizaciones Latinoamericanas, 6, (11), 1-5, 2014.

WALSH, C. Interculturalidad crítica y educación intercultural. En: VIAÑA, J.; TAPIA, L. y WALSH, C. (Eds.), Construyendo Interculturalidad Crítica, p. 75-96, Bolivia: Instituto Internacional de Integración del Convenio Andrés Bello, 2010.

WILLIAMSON, G.; FLORES, F. Estado del arte de la Educación Intercultural Bilingüe en Chile, 1990-2013. Ediciones Universidad de la Frontera Facultad de Educación, Ciencias Sociales y Humanidades, 2015.

Texto recibido el 26 de noviembre de 2018. Texto aprobado el 19 de marzo de 2019. 